\title{
Combining the Preparation of Oligonucleotide Arrays and Synthesis of High-Quality Primers
}

\author{
J an Weiler ${ }^{1}$ and J örg D. Hoheisel \\ Molecular-Genetic Genome Analysis, Deutsches Krebsforschungszentrum, Im Neuenheimer Feld 506, \\ D-69120 Heidelberg, Germany
}

Received J une 27, 1996

Based on the oligomer-chip technology, oligonucleotide arrays were synthesized directly on polypropylene sheets by a modified phosphoramidite chemistry using $\beta$-eliminating nucleobase-protecting groups in combination with a succinate solid-phase linker. This method decouples the oligonucleotide deprotection from the support cleavage procedure, in contrast to standard phosphoramidite chemistry. In addition to being reliable substrates for hybridization experiments, the arrays also serve as source for the isolation of individual oligonucleotides. Technically, this allowed for a direct control of the quality of the arrayed oligomers. The released compounds were sufficient in amount and purity to work without further purification in PCR and DNA-sequencing reactions, with the results being identical to controls with commercially obtained primer molecules. Consequences for oligomer-chip hybridization procedures, the applicability of such hybrid-function arrays in, for example, diagnostics or comparative biology, and developments toward parallel primer synthesis are discussed. $\odot 1996$ Academic Press, Inc.

Initiated by the combination of solid-phase technology and the phosphoramidite chemistry introduced by Beaucage and Caruthers (1), there has been much progress in the automation of DNA synthesis. Today, the preparation of synthetic ol igonucl eotides needed in biological, biomedical, and physical applications is usually executed with commercial, automated DNA synthesizers. These machines produce oligonucleotides in the nanomole up to micromole range. However, over the past few years, there have been two diametrical tendencies with regard to oligomer synthesis for biomedical

\footnotetext{
${ }^{1}$ To whom correspondence should be addressed. Fax: +49-6221424682. E-mail: j.weiler@dkfz-heidelberg.de.
}

purposes. For clinical applications like the antisense strategy (reviewed in Ref. 2), gram or even kilogram amounts of oligonucleotides are required, prompting efforts on an up-scaling of synthesis yields (e.g., 3). For many applications in molecular biology, on the other hand, notably DNA characterization and screening by hybridization and enzymatic assays like the polymerase chain reaction (PCR) and DNA sequencing, oligomer quantities in the picomole range are usually an adequate amount, but larger numbers of oligonucleotides are needed. Again, this has led to procedures aiming at the simultaneous production of different oligonucleotides in small quantities (e.g., 4, 5).

More recently, another consequential technology in molecular biology was devel oped using ordered arrays of oligonucleotides to serve as analytic and diagnostic tools for DNA analyses (6-8). This oligomer-chip technology has a huge potential to play an important role in many aspects of genome analysis (for a review see Ref. 9). Technically most challenging is the sequencing by hybridization $(\mathrm{SBH})^{2}$ strategy, whereby the sequence of an unknown nucleic acid is reconstructed from its hybridization binding pattern on a matrix which contains a comprehensive set of short oligonucleotide sequences (e.g., all 65,536 octamers).

During our work concerned with developments toward a practical application of the SBH technique, such as the ordering and selection of DNA fragments suitable for SBH analysis $(10,11)$, for example, or the sequence-independent leveling of oligomer binding stabilities (12), it was apparent to us that such oligomer chips by design would be an ideal tool not only for screening procedures but also for the synthesis of

\footnotetext{
${ }^{2}$ Abbreviations used: SBH, sequencing by hybridization; TOTU, O-[(ethoxycarbonyl)cyanomethylenamino]- $N, N, N^{\prime}, N^{\prime}$-tetramethyluronium tetrafluoroborate; DBU, 1,8-diazabicyclo-(5.4.0)-undec-7ene; NPE, 2-nitrophenylethyl; NPEOC, 2-(4-nitrophenyl)ethoxycarbonyl; POM, polyoxymethylene; DMF, dimethylformamide; THF, tetrahydrofuran.
} 
primer molecules, if quantity and purity of each individual oligomer would be sufficient for biological reactions. For this purpose, a modification of the synthesis chemistry was needed that would allow the use of the oligomer grids both for hybridization experiments and for the isolation of the individual oligomers. To this end, the deprotection step after completed oligonucleotide synthesis had to be disconnected from the step of cleaving the oligonucleotides off the support medium, with both manipulations taking place at high efficiency. Here we describe the establishment of such a synthesis procedure and demonstrate the suitability of the released oligonucleotides in amount and purity for PCR amplification and DNA sequencing.

\section{MATERIALS AND METHODS}

Reagents. O-[(Ethoxycarbonyl)cyanomethylenamino]- $\mathrm{N}, \mathrm{N}, \mathrm{N}^{\prime}, \mathrm{N}^{\prime}$-tetramethyluronium tetrafluoroborate (TOTU), 1,6-bis-(methylamino)-hexane, p-nitrophenylchloroformate, N-methylmorpholine, 1,8-diazabicydo(5.4.0)-undec-7-ene (DBU), N-methylimidazole, t-butyl hydroperoxide, and solvents were purchased from Fluka and used without additional purification. Amino-functionalized polypropylene sheets wereobtained from Beckman; 5' -O-DMT-2' -deoxynudeoside-3'-O-( $\beta$-cyanoethyl)-N,N diisopropyl-phosphoramidites protected by 2-nitrophenylethyl(NPE) and 2-(4-nitrophenyl)ethoxycarbonyl (NPEOC) functions was from Chemogen (Constance, Germany). If not indicated otherwise, ol igonudeoti des used as hybridization probes or as controls in enzymatic reactions were purchased from MWG Biotech (Ebersberg, Germany). [ $\left.\gamma-{ }^{32} \mathrm{P}\right]$ ATP $(10 \mathrm{mCi} / \mathrm{ml}), \mathrm{T} 4$ polynucleotidekinase, and buffers for labeling oligomers at their 5 -end were from Amersham and New England Bidabs, respectively.

Chamber for the synthesis of oligonud eoti de arrays. For the initial experiments, a simple polyoxymethylene (POM) block was constructed containing eight channels of $1 \mathrm{~mm}$ depth, $70 \mathrm{~mm}$ length, and $4 \mathrm{~mm}$ width each, with two holes at both ends for connection to the inlet and outlet of a commercial DNA synthesizer. The polypropylene film was held in place by a silicon seal and a lid of perspex screwed to the POM basis (Fig. 1). For perfect sealing, additional pressure was applied to the whole apparatus by a clamp. By removing the polypropylene sheet after a singleor several cycles, rotation by $90^{\circ}$, and continued synthesis, this simple and small instrument al lowed the synthesis of up to 64 oligomers.

Preparation of methylamino-modified polypropylene support. In a typical experiment, aminated polypropylene sheets $\left(8 \times 8 \mathrm{~cm}^{2}\right)$ were gently shaken in a mixture of $25 \mathrm{ml}$ dry dichloromethane, $25 \mathrm{ml}$ dry dioxane, $0.2 \mathrm{~g}$ p-nitrophenylchlor oformate, and $160 \mu$ l triethylamine for $2 \mathrm{~h}$ at room temperature. After washing with dichloromethane, the sheets were shaken in a 1:1 mix-

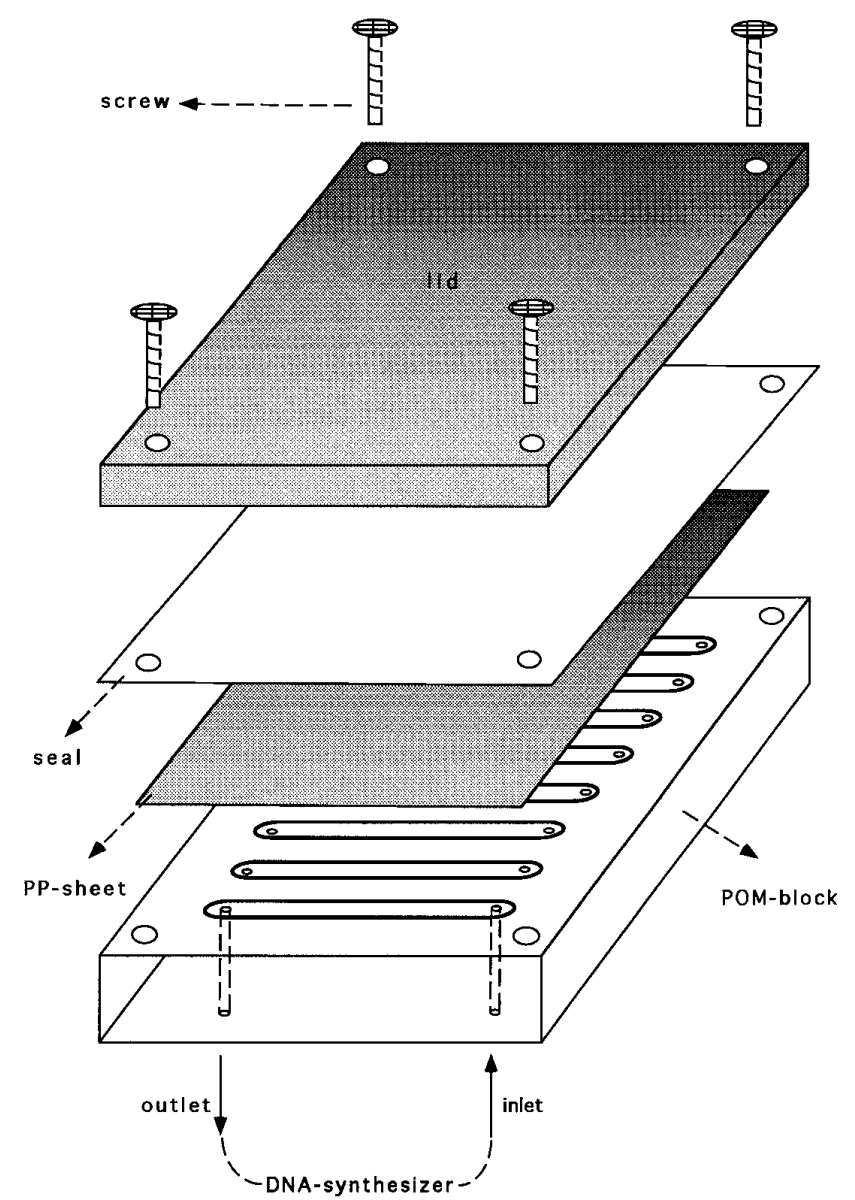

FIG. 1. Drawing of the reaction chamber used for oligonucleotide synthesis on polypropylene sheets. The device is actually square in format to allow $90^{\circ}$ rotations of the polypropylene sheet between synthesis steps.

ture of pyridine and acetic anhydride for $2 \mathrm{~h}$, capping amino groups remaining on the surface. Subsequently, the sheets were washed with dichloromethane and taken up in $50 \mathrm{ml}$ acetonitrile or dimethylformamide (DMF); $0.2 \mathrm{ml}$ 1,6-bis-(methylamino)-hexane was added and the mixture was shaken at $40^{\circ} \mathrm{C}$ for $48 \mathrm{~h}$. After washing successively with DMF, methanol, and acetone, the methylamino-modified polypropylene was dried and stored at $4^{\circ} \mathrm{C}$.

Derivatization of methylamino-modified support with nucleosides. 3'-O-Succinate derivatives of protected nucleosides ( $d A^{\mathrm{NPEOC}}, \mathrm{dC}^{\mathrm{NPEOC}}, \mathrm{dG}^{\mathrm{NPEOC} / \mathrm{NPE}}, \mathrm{dT}$, and fluorescein-labeled dC [= dCf; Ref. 13]) were prepared by the method of Kierzek et al. (14) and were loaded to the methylamino-modified polypropylene after mounting a sheet in the synthesis chamber. Five milligrams of the desired nucleoside-3'-succinate was mixed with $25 \mu \mathrm{l} \mathrm{N}$-methylmorpholine and $10 \mathrm{ml}$ acetonitrile, before adding $4 \mathrm{mg}$ of TOTU. The bottle with the mixture was immediately connected to the DNA synthe- 


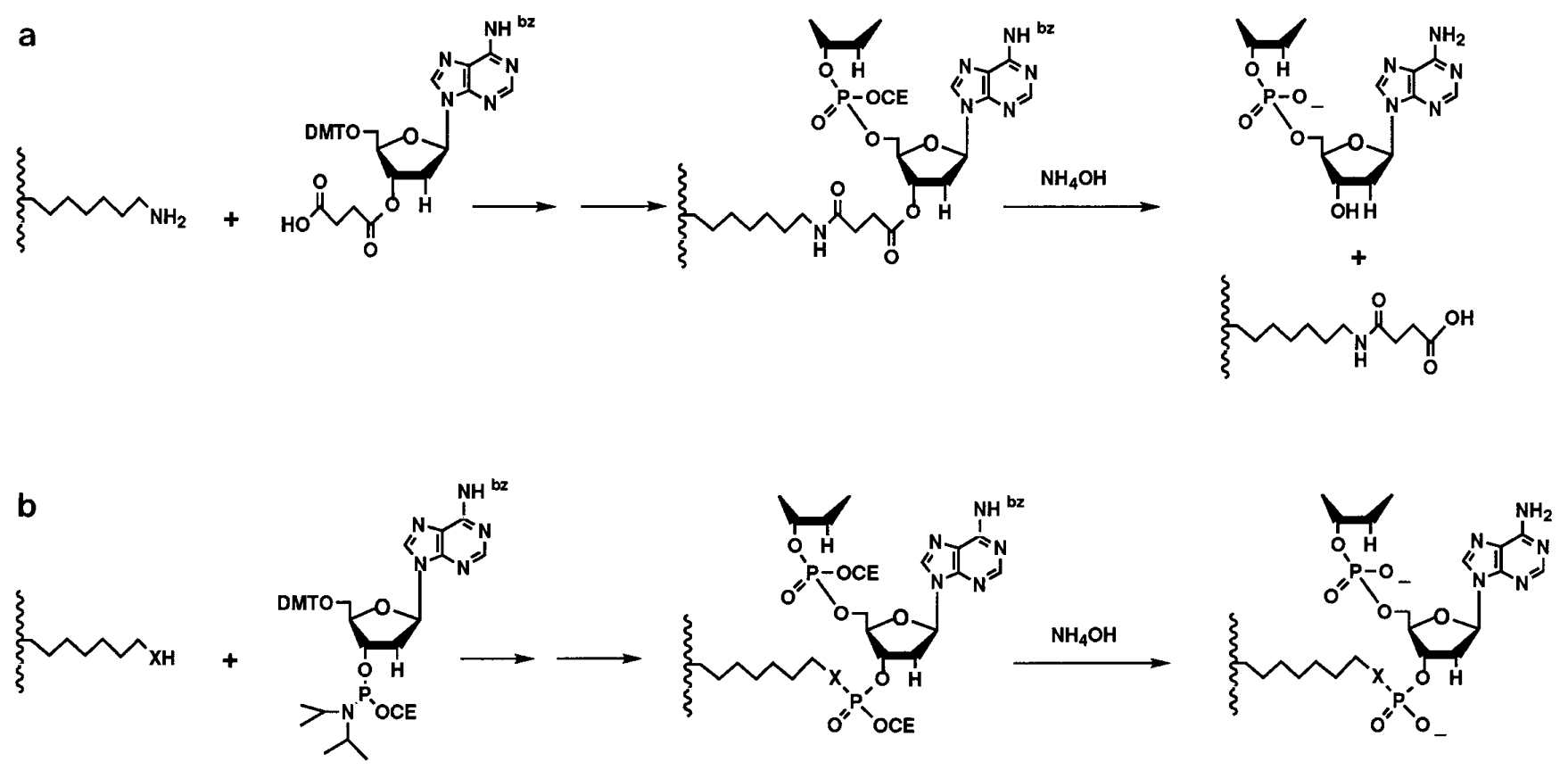

FIG. 2. Probe attachment chemistries and deprotection by ammonia $\left(\mathrm{NH}_{4} \mathrm{OH}\right)$ used for standard synthesis of free oligonucleotide (a) and the preparation of oligonucleotide arrays (b); $\mathrm{X}=\mathrm{O}(16) ; \mathrm{X}=\mathrm{NH}$ (17).

sizer and a simple preprogrammed cycle was activated: first the rows were rinsed with the reaction mixture and the reaction was kept for $30 \mathrm{~min}$; then, the reagents were flushed out with argon and the rows were washed with acetonitrile. To block residual methylamino groups on the polypropylene, capping solution as described below was applied. Alternatively, the derivatized polypropylene membranes were removed from the chamber and shaken in a mixture of $10 \mathrm{ml}$ acetic anhydride, $10 \mathrm{ml}$ dry pyridine, and $1 \mathrm{ml} \mathrm{N}$-methylimidazole for $2 \mathrm{~h}$ in a polypropylene box. After successive washing with DMF, methanol, and acetone, the sheets were dried and stored at $4^{\circ} \mathrm{C}$ until usage.

Oligonucleotide synthesis. Oligonucleotide synthesis on polypropylene sheets was carried out as detailed in Table 1. After completed synthesis, the sheet was removed from the chamber and shaken in $1 \mathrm{~m}$ DBU in acetonitrile in a polypropylene box at $40^{\circ} \mathrm{C}$ overnight for oligomer deprotection. The membrane was washed with acetonitrile and acetone, before being used for hybridization experiments or cleavage of the oligomers, respectively. No deterioration of experimental results was observed even after storing the oligomer arrays over several weeks at room temperature.

Hybridization with oligonucl eotideprobes. Oligonucleotide probes were end-labeled with [ $\gamma^{32}$ P]ATP under standard conditions (15). The oligomer arrays were prehybridized in $600 \mathrm{~mm} \mathrm{NaCl}, 60 \mathrm{~mm}$ sodium citrate, $\mathrm{pH} 7.5,7.2 \%$ sodium $\mathrm{N}$-lauroylsarcosine for $1 \mathrm{~h}$ and then incubated in $10 \mathrm{ml}$ of the same solution containing about 1 Mcpm radioactively labeled oligomer probe (concentration $=6 \mathrm{pmol} / \mathrm{ml}$ ) for $18 \mathrm{~h}$ at $4^{\circ} \mathrm{C}$. After washing for $30 \mathrm{~min}$ at $4^{\circ} \mathrm{C}$, autoradiography was at $-70^{\circ} \mathrm{C}$ using intensifying screens. Probes were stripped off the sheets by incubation in hybridization buffer at $65^{\circ} \mathrm{C}$ for $3 \mathrm{~h}$.

Releasing oligonucleotides from the polypropylene support. For cleaving off individual oligonucleotides, the desired square of an oligomer array on a polypropylene sheet was cut out with a key punch machine. After incubation in 30\% aqueous ammonia for $2 \mathrm{~h}$, the re leased oligonucleotide product was lyophilized and used for PCR and DNA sequencing without further purification.

\section{RESULTS}

Usually, for oligonucleotide synthesis the common $\beta$-cyanoethyl phosphoramidite chemistry is used: the phosphorus of the monomer building blocks is protected with a $\beta$-cyanoethyl function, and acyl functionalities like benzoyl-(dA $\left.{ }^{b z}, d C^{b z}\right)$, acetyl-( $\left.d C^{a c}\right)$, or isobutyryl-groups ( $\mathrm{dG}^{\mathrm{ibu}}$ ) are used for nucleobase blocking. After completion of the oligonucleotide chain elongation process, the protecting groups are removed nucleophilically by incubation in concentrated aqueous ammonia at $60^{\circ} \mathrm{C}$ (Fig. 2a). The difference between the synthesis of free oligonucleotides in commercial machines and the construction of oligomer arrays $(16,17)$ is the anchoring to the solid support at the $3^{\prime}$-terminal 

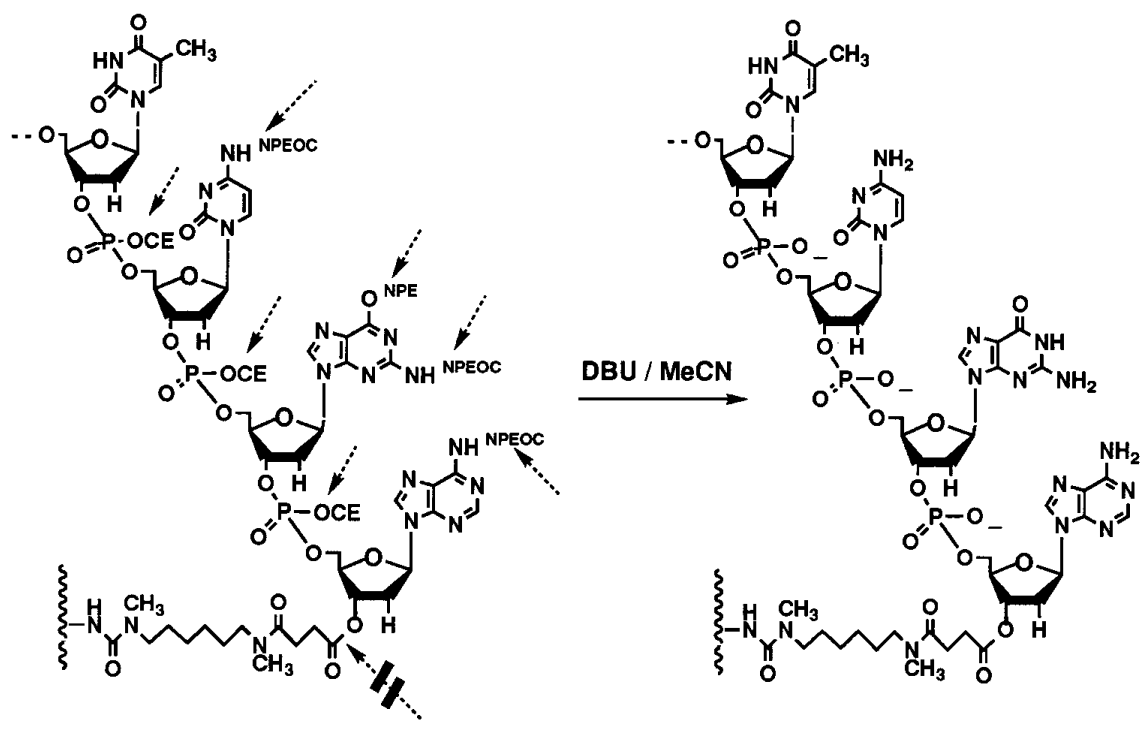

Option l: Hybridization experiments on oligonucleotide arrays

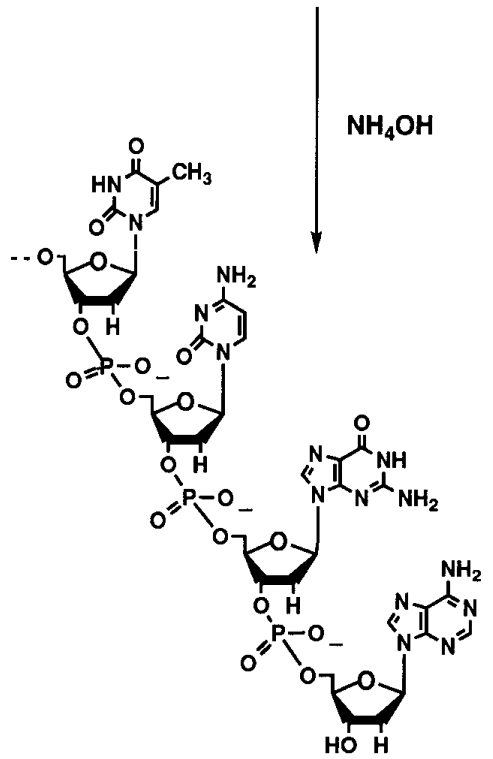

Option II: Release of DNA-primers for PCR or sequencing

FIG. 3. Separation of nucleobase deprotection of solid-phase-bound oligonucleotides from support cleavage. Application of the NPE/NPEOC strategy allows alternative use of the oligomer arrays for either hybridization experiments (option I) or as a source for the isolation of individual primer molecules (option II).

nucleoside. While for the former an ester bridge like the succinatelinker is intentionally cleaved simultaneous to the biopolymer deprotection, the arrayed oligonucleotides must remain attached to the solid phase after the deprotection process for hybridization experiments. In earlier experiments, this was achieved by linkage via a stable phosphoester (16) or phosphoramidate (17) bond (Fig. 2b).

For the preparation of oligonucleotide hybrid arrays that combine usage in hybridization screening experi- ments with the capability of cleaving the oligonucleotides off the support, to be used as probes or primer molecules for enzymatic reactions like PCR or DNA sequencing, for example, nucleobase deprotection had to be decoupled from the release reaction (18), with nevertheless stable binding during oligomer synthesis and any subsequent hybridization experiment. This goal was reached by using the common succinate linker between the 3'-terminal nucleosideand the solid phase in combination with an alternative phosphoramidite 


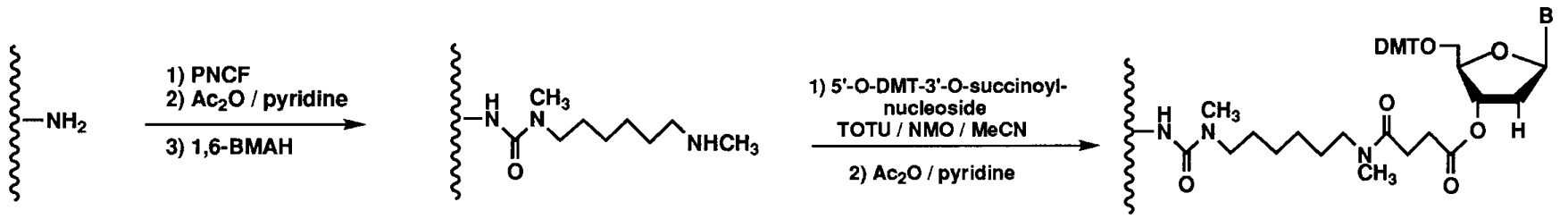

PNCF = p-nitrophenyichloroformate

1,6-BMAH = 1,6-bis-(methylamino)-hexane
NMO = n-methylmorpholine

$B=d A^{\text {NPEOC }}, d G^{\text {NPE/NPEOC }}, d C^{\text {NPEOC }}, d T, d C^{\text {flu }}$

FIG. 4. Activation of the methylamino-modified polypropylene sheets and coupling of appropriately protected nucleosides.

approach, the so-called "NPE/NPEOC strategy" (19), which on CPG and polystyrene supports had led to oligonucleotide products of high purity $(20,21)$. This synthesis strategy uses the $\beta$-eliminating base-protecting groups NPE and NPEOC. These functions allow the deprotection of the biopolymer by thestrong but nonnucleophilic base DBU while the oligonucleotide remains attached to the polypropylene (Fig. 3).

\section{Surface Modification}

The chemistry used for synthesis required an anchoring structure between the oligonucleotides and the aminated polypropylene sheets which is cleavable under nucleophilic conditions, i.e., by treatment with concentrated aqueous ammonia, but remains stable under the nonaqueous DBU deprotection conditions. In accordance with results reported earlier (22), we found it necessary to introduce an alkylamino-modified spacer between the primary amino group of the polypropylene sheet and the succinate linkage to meet these requirements (Fig. 4). The yield of this activation step was determined by p-nitrophenolateliberation (23) to range between 30 and $90 \mathrm{pmol} / \mathrm{cm}^{2}$.

\section{TABLE 1}

Protocol Used for the Production of Oligonudeotide Arrays on Polypropylene Membranes U sing the Eight-Channel Synthesis Chamber Connected to an ABI-392 DNA Synthesizer

\begin{tabular}{llr}
\hline Step & Reagents or solvent & Time (s) \\
\hline Washing & Acetonitrile & 30 \\
Detritylation & 3\% TCA in dichloromethane & 80 \\
Washing & Acetonitrile & 210 \\
Coupling & 75 mM phosphoramidite + & 40 \\
& $\quad 0.5$ M tetrazole in & \\
Washing & acetonitrile & 30 \\
Capping $(I+$ II) & Acetonitrile & 50 \\
Oc 2 /N-methylimidazole in & 130 \\
Washing & acetonitrile & 120 \\
Total & 1 M t-butyl hydroperoxide in & 690 \\
\hline
\end{tabular}

\section{In Situ Oligonud eoti de Synthesis}

Initially oligomer synthesis on the polypropylene sheets with the NPE/NPEOC nucleoside derivatives was carried out under running standard phosphoramidite chemistry protocols. In subsequent PCR, however, such primers could not equal the performance obtained with primer molecules of identical sequence that were synthesized as a control on CPG material using the same chemistry. Intensive studies of the synthetic conditions indicated that mainly the tetrahydrofuran (THF) solvent included in standard capping and oxidation solutions was responsible for low coupling yields and/or product contamination on the polypropylene support. Most probably, a swelling of the polypropylenefilm in the presence of THF (R. Matson, personal communication) obstructed the complete removal of unreacted reagents, thus leading tolargely decreased coupling yields. Much better results were achieved when

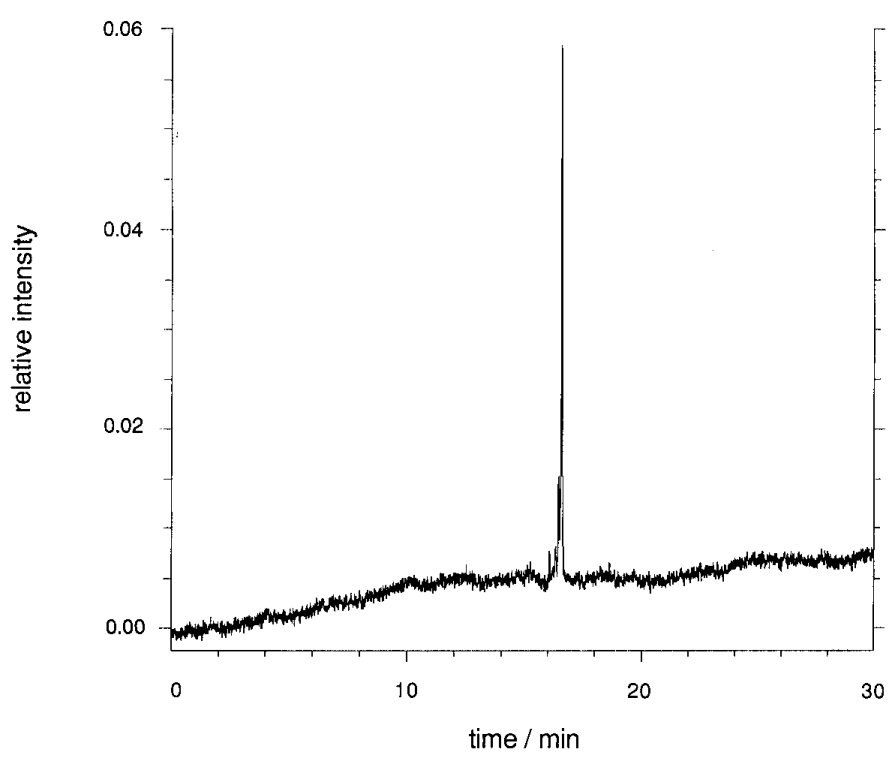

FIG. 5. Capillary electropherogram of a 15-mer oligonucleotide that had been synthesized on a polypropylene sheet. Electrophoresis was carried out in a gel-filled tube, and detection was via a fluorescein label at the $3^{\prime}$-terminal base. 
a

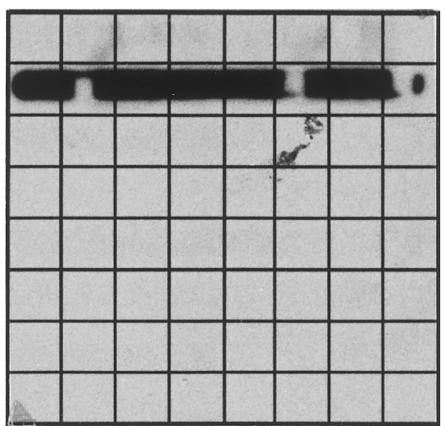

A 5'-TCACTATAGgGaATtCGaGCTCGgta-X

B 5-GCTTGCATGCCTGCAGGTCGACTCT-X

C 5 -ITTTTTTTT-X

D -

E 5 -TTTTTTTTTTT-X

F 5'-CGAGCTCGGTA- $x$

G $\quad 5$ -

H 5'-AGGTCGACTCT-X

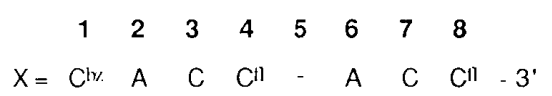

c 5'-GCCGTCGTT-3'

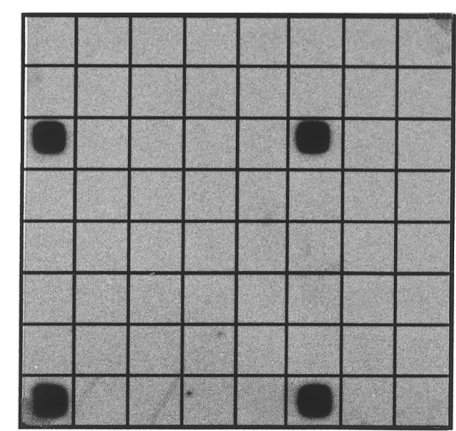

A 5'-TAATACGACTCACTATAGGG- $X$

B 5-GCTTGCATGCCTGCAGG-X

C 5'-GTAAAACGA-X

D $5^{\prime} \cdot x$

E $5^{\prime}-\mathrm{X}$

F 5-TAATACGACTCACTATAGGG- $X$

G 5'-GCTTGCATGCCTGCAGG-X

H 5'-GTAAAACGA-X b 5'-GAAAAAAAAAAA-3
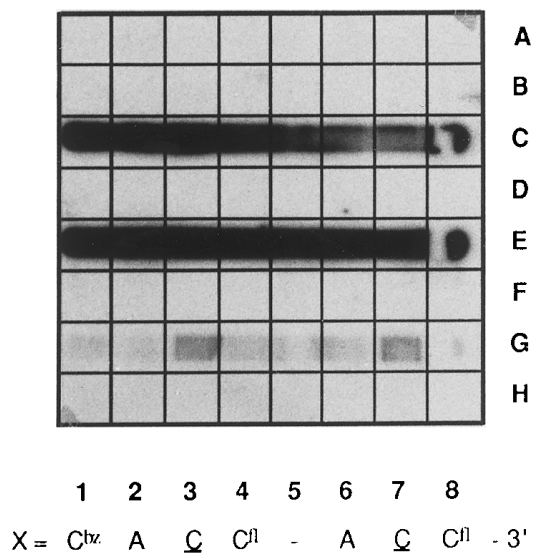

d

5'-GTCGACCTG-3'

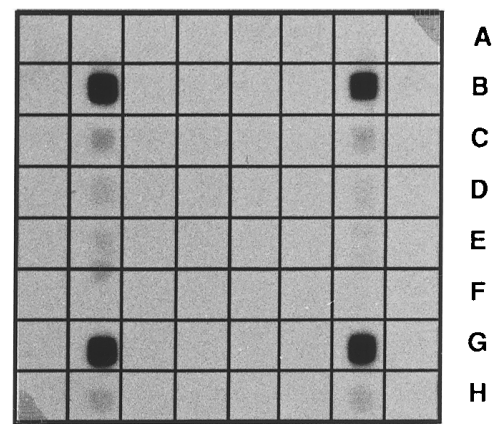

\begin{tabular}{cc|cccccccc}
$\mathbf{1}$ & 2 & 3 & 4 & 5 & 6 & 7 & 8 & \\
$\mathrm{X}=\mathrm{C}$ & $\mathrm{T}$ & $\mathrm{A}$ & $\mathrm{T}$ & - & $\mathrm{C}$ & $\mathrm{T}$ & $\mathrm{A}$ & \\
$\mathrm{G}$ & $\mathrm{C}$ & $\mathrm{A}$ & $\mathrm{T}$ & - & $\mathrm{G}$ & $\mathrm{C}$ & $\mathrm{A}$ & \\
$\mathrm{G}$ & $\mathrm{G}$ & $\mathrm{T}$ & $\mathrm{T}$ & - & $\mathrm{G}$ & $\mathrm{G}$ & $\mathrm{T}$ & \\
$\mathrm{C}$ & $\mathrm{A}$ & $\mathrm{T}$ & $\mathrm{T}$ & - & $\mathrm{C}$ & $\mathrm{A}$ & $\mathrm{T}$ & \\
$\mathrm{A}$ & $\mathrm{C}$ & $\mathrm{C}$ & $\mathrm{T}$ & - & $\mathrm{A}$ & $\mathrm{C}$ & $\mathrm{C}$ & \\
$\mathrm{G}$ & $\mathrm{T}$ & $\mathrm{G}$ & $\mathrm{T}$ & - & $\mathrm{G}$ & $\mathrm{T}$ & $\mathrm{G}$ & \\
$\mathrm{T}$ & $\mathrm{C}$ & $\mathrm{A}$ & $\mathrm{T}$ & - & $\mathrm{T}$ & $\mathrm{C}$ & $\mathrm{A}$ & \\
$\mathrm{G}$ & $\mathrm{T}$ & $\mathrm{G}$ & $\mathrm{C}$ & - & $\mathrm{G}$ & $\mathrm{T}$ & $\mathrm{G}$ & \\
& $\mathrm{A}$ & $\mathrm{C}$ & & - & & $\mathrm{A}$ & $\mathrm{C}$ & $-3^{\prime}$
\end{tabular}

FIG. 6. Typical hybridizations to oligomer arrays on polypropylenemembranes. (a, b) Four different starting nucleosides, dC ${ }^{N P E O C}$ (columns 3 and 7), $\mathrm{dA}^{\mathrm{NPEOC}}$ (columns 2 and 6), $\mathrm{dC}^{\mathrm{bz}}$ (column 1), and $\mathrm{dC}^{\mathrm{fl}}$ (columns 4 and 8), were coupled to a polypropylene sheet using the eightchannel synthesis device. The sheet was removed from the chamber and rotated $90^{\circ}$. Subsequently, seven different oligonucleotides were synthesized $(\mathrm{A}-\mathrm{H})$ creating 49 squares containing 28 different sequences. One channel was left empty in both dimensions as a control (lanes 5 and D). After oligomer deprotection, some squares (e.g., B2, B6, B8, C8, and E8) were cut out for analyses. The remaining sheet was hybridized with (a) the 9-mer d(CTATAGTGA) and (b) the 12-mer d(GA 11 ). Complementary sequences are underlined. In (c) and (d), the sheet had been turned $90^{\circ}$ in between synthesis steps. Nonamers whose sequences cover breakpoints of synthesis were hybridized.

the standard capping reagents (capping I: THF/2,6-lutidine/A $\mathrm{C}_{2} \mathrm{O}$, 8:1:1; capping II: N-methylimidazole/THF, 16:84) were replaced by a $1: 1$ mixture of $\mathrm{Ac}_{2} \mathrm{O}$ and acetonitrile (capping I) and a 2:8 mixture of $\mathrm{N}$-methylimidazole and acetonitrile (capping II). Furthermore, instead of iodine, a 1 m solution of t-butyl hydroperoxide in acetonitrile was applied for oxidation. The final protocol used for oligonucleotide synthesis on polypropylene is shown in Table 1. Approximately $300 \mu$ l of 75 mM phosphoramidite solutions was applied for each coupling step so that the coupling reagents were in great excess over the growing nucleotide chain on the 


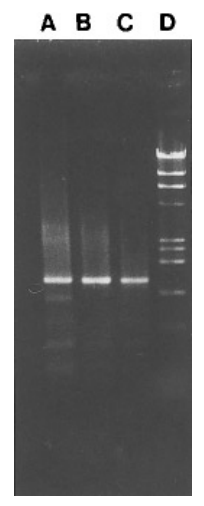

FIG. 7. $P C R$ amplification of a plasmid insert. Both commercial primers were used for the reaction run in lane A. In lanes B and C, either of the primer molecules had been substituted for by oligonucleotide isolated from $0.16 \mathrm{~cm}^{2}$ of a polypropylene sheet. Marker (lane D) is HindlII-digested $\lambda$-DNA and plasmid pUC18 cut with Fspl.

polypropylene surface. This new kit of reagents for DNA synthesis also succeeded in producing high-quality oligonucleotides in common solid-phase syntheses on CPG support in the $0.2-\mu \mathrm{mol}$ scale. Examination of the products by analyses based on HPLC separation and capillary electrophoresis did not find any differences in quality - such as the occurrence of base modifications - compared to standard synthesis protocols.

\section{Oligomer Quality}

Oligonucleotides synthesized on polypropylene were deprotected by a DBU treatment and subsequently removed by ammonia from the surface of the support for analyses of purity and quantity. The appropriate piece of a polypropylene film was cut out, and the oligomer was released by a treatment in $30 \%$ aqueous ammonia. Lyophilized, the DNA was taken up in water. Oligonucleotides of up to $29 \mathrm{bp}$ in length were labeled either at the beginning of an in situ synthesis at the eventual 3 '-terminal base by incorporation of a fluorescein-labeled cytosine (13) as the starting nucleoside or by transfer of a radioactive phosphate group to the 5 '-end of a released oligonucleotide using T4 polynucleotide kinase. Analysis of molecule length was by direct fluorescence detection after capillary electrophoresis in a Beckman P/ACE 5000 machine (e.g., Fig. 5) or autoradiography subsequent to polyacrylamide gel electrophoresis (not shown), respectively. With the final, modified synthesis protocol (Table 1), most of the oligonucleotide product was found to be full-length molecules indicating a stepwise coupling efficiency of more than $98.5 \%$. From an evaluation of the signal intensities detected in the same experiments, the amount of released oligonucleotide probe could be calculated to be more than $25 \mathrm{pmol} / \mathrm{cm}^{2}$ polypropylene. This value is in good agreement with the 30- to 90-pmol range determined for the initial activation step prior to synthesis.

\section{Hybridization Experiments}

First, to confirm the stability of the succinate linker during the DBU deprotection and hybridization conditions and, second, to test the selectivity of hybridizations to the polypropylene matrices, oligomer arrays were used for hybridization experiments, in some cases after individual oligomers had been removed for other analyses. Each sheet was briefly washed in hybridization buffer prior to hybridization experiments with radioactive probes. Duplex formation was detected by autoradiography. Typical results on test grids are presented in Fig. 6. The succinate linkage was found to be stable during DBU deprotection and hybridization and stripping procedures. Arrays were used in five subsequent hybridization experiments without apparent loss of signal. Oligomer probes were applied that were complementary to either the $5^{\prime}$-end (Fig. $6 \mathrm{a}$ ) or the 3 '-end (Fig. 6b) of individual oligonucleotides on the grid. Positive signals by the latter proved the accessibility to hybridization of the most 3'-position of a surface-bound oligonucleotide, the former confirmed the presence of the $5^{\prime}$-end. The equivalent signal intensities detected with both probes suggested high coupling yields during synthesis, thus confirming the results of the more stringent quality checks on released oligomers. One base pair mismatch selectivity was achieved by hybridizing to target sequences as short as hexamers, even if there was sequence repetition and the difference between the corresponding terminal nucleotide was only a substitution to the nucleobase (Fig. 6b).

\section{PCR and Sequencing Applications of Released Oligonucleotides}

For enzymatic reactions, oligonucleotide primers were removed from the polypropylene and taken up in water without further purification. PCR on recombinant plasmid pTZ18R was done under standard conditions as described in detail earlier (11). Primers were 26- and 29-mers binding to the vector directly adjacent to either side of the insert DNA. An oligonucleotide amount equivalent to an area of $0.16 \mathrm{~cm}^{2}$ of the polypropylene surface was used in reactions of $25 \mu \mathrm{l}$. Twotemperature PCR was done, primer annealing and extension at $68^{\circ} \mathrm{C}$ and strand denaturation at $95^{\circ} \mathrm{C}$. On agarose gels, the products were compared to the results obtained with commercial primer molecules used at a concentration of $1 \mu \mathrm{M}$. No significant difference in quality or quantity of the PCR products could be observed (e.g., Fig. 7).

The ultimate test of the coupling efficiency during synthesis, and hence oligonudeotide quality, is its use as primer in an enzymatic sequencing reaction. Even relatively small contaminations by shorter side products would lead to extra bands in the sequencing gel. 

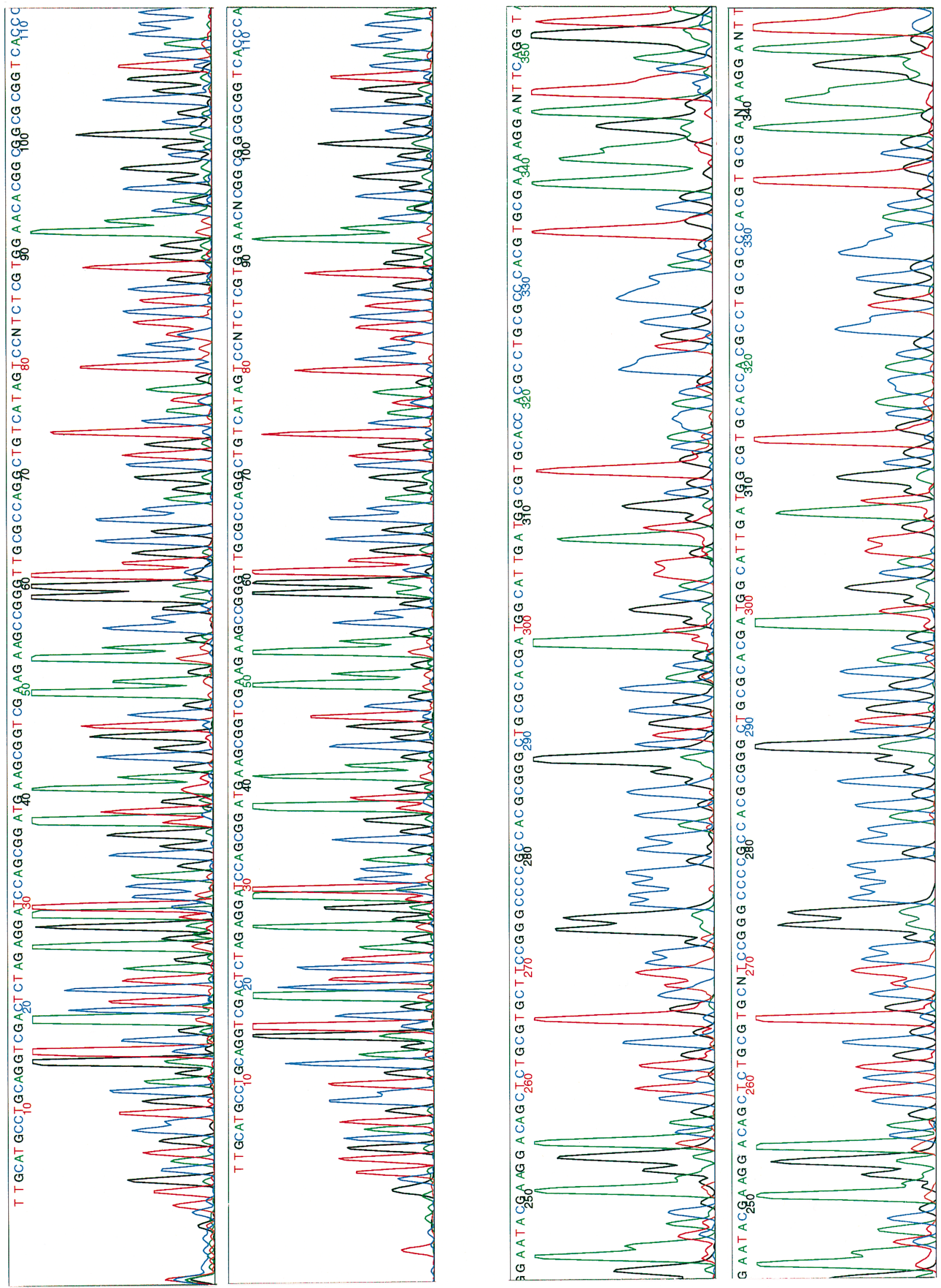

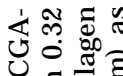

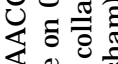

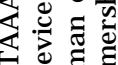

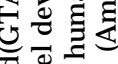

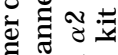

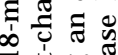

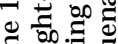

등

o

응.

웅중

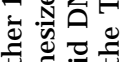

形实可

这

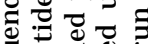

政

mे 흔해

m o ठd

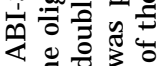

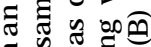

등

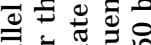

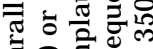

呕

등

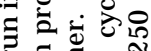

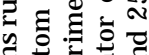

흘ㅎㅇㅎㅁㅇㅝ

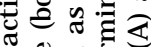

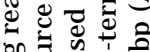

둥 능

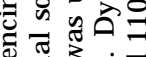

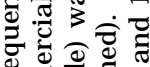

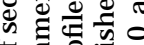

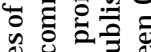

흔

흥

Q

过 的西.

政

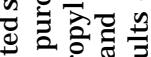

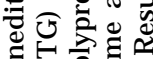

5 응히잉

$\infty$ 论它

ज्ञ 
In a typical experiment, the 18-mer "forward" primer d(GTAAAACGACGGCCAGTG) of the bluescript plasmid was isolated from polypropylene and used in sequencing reactions. Oligomer taken from $0.32 \mathrm{~cm}^{2}$ was used per standard cycle sequencing reaction. The quality of the sequencing profiles was compared to reactions using commercial primer. Again, no apparent difference in performance could be detected (Fig. 8).

\section{DISCUSSION}

A modified phosphoramidite chemistry on a polypropylene membrane as solid support was established that not only allows oligonucleotide arrays to be used as substrates in hybridization experiments but also offers the opportunity to separate each compound from the synthesized oligomer grid. With regard to the SBH technology, this provides the means for a direct quality assessment of the oligomers synthesized on the array, rather than to use results of hybridization experiments for such evaluation. Recent investigations (24) showed that the reliability of hybridizations to arrays of short oligonucleotides depends strongly on the quality of the array-bound oligomers. Hence, both the high-quality oligonucleotides on the chip and the independent control mechanism provided by the presented technique are paramount for accurate SBH analysis.

Some further improvements concerning the chemistry are currently being investigated: preliminary experiments indicate, for example, that the time of oligomer production could be shortened drastically, in particular by abbreviating the period of the DBU deprotection process from 12 to $1 \mathrm{~h}$ at higher reaction temperatures. Also, technical aspects require further development. The eight-channel device had only been designed as a very simple tool for initial analyses. However, key issues could be tested, such as the flow of chemicals through the channels or the sealing mechanism. From this basis, construction of much more re fined instruments for different applications is underway. Another problem that is being addressed is the control of the synthesis cycles. Although a commercial machine was sufficient on a small scale, modified instruments are needed for automatically serving larger arrays. Concomitant, technical advances are in progress to reduce substantially the consumption of re agents by adapting the tubing and vent system toward a reagent delivery system that is more effective than a standard DNA synthesizer.

The complete and efficient separation of nucleobase deprotection and release from the solid support, the stability of the succinate linker during hybridization, and the high quality of the oligomer molecules suggest a combined use of the arrays both as an instrument for screening experiments and as source for oligonucleotide primers. Thereby, an oligomer could first serve as a detector molecule, immediately followed by its use for an isolation of the appropriate DNA fragment by PCR or a direct tag sequence analysis of the region. Also, oligonucleotides identified by hybridization of a given DNA could in turn be used as probes for the identification of homologous fragments, for example, an approach that would simplify and speed up comparative genome analyses. F or some applications, it might be necessary to introduce a stretch of unspecific bases at the $3^{\prime}$ terminus in order to ensure an enzymatic extension of the molecules by a polymerase. Due to combinatorial constraints, only a moderate number of sequence variations can be synthesized in parallel on a device of a type as depicted in Fig. 1. Nevertheless, such kind of array is of interest for diagnostic applications, for example. Moreover, the results suggest that ultimately even a larger number of independent oligonucleotides could be generated by an appropriately adjusted device. This could make techniques that require small amounts of different oligonudeotides such as primer walking sequencing even more functional.

\section{ACKN O WLEDGMENTS}

We thank Sandra Schwarz for excellent technical assistance, Stephen Case-Green and Edwin Southern for help in establishing the technique of oligonucleotide array construction and useful discussions, Robert Matson of Beckman Instruments for providing aminated polypropylene sheets and related information, Werner Fleischer and Wolfgang Schütz for generously letting us work with their DNA-synthesizer, and Achim Karger for analyses by capillary electrophoresis. The work was funded by the Deutsche F orschungsgemeinschaft.

\section{REFERENCES}

1. Beaucage, S. L., and Caruthers, M. H. (1980) Tetrahedron Lett. 22, 1859- 1862 .

2. Crooke, S. T. (1995) Hematol. Pathol. 9, 59- 72.

3. Wright, P., Lloyd, D., Rapp, W., and Andrus, A. (1993) Tetrahe dron Lett. 34, 3773-3776.

4. Sindelar, L. E., and J aklevic, J . M. (1995) Nucleic Acids Res. 23, 982- 987.

5. Lashkari, D. A., Hunicke-Smith, S. P., Norgren, R., Davis, R. W., and Brennan, T. (1995) Proc. Natl. Acad. Sci. USA 92, 79127915.

6. Khrapko, K., Lysov, Y., Khorlyn, A., Shick, V., Florentiev, V., and Mirzabekov, A. (1989) FEBS Lett. 256, 118- 122.

7. Southern, E. M., Maskos, U., and Elder, J . K. (1992) Genomics 13, 1008- 1017.

8. Caviani-Pease, A. C., Solas, D., Sullivan, E.J ., Cronin, M.J., Holmes, C. P., and Fodor, S. P. A. (1994) Proc. Natl. Acad. Sci. USA 91, 5022-5026.

9. Mirzabekov, A. D. (1994) Trends Biotechnol. 12, 27- 32.

10. Hoheisel, J . D. (1994) Trends Genet. 10, 79- 83.

11. Scholler, P., Karger, A. E., Meier-Ewert, S., Lehrach, H., Delius, H., and Hoheisel, J. D. (1995) Nucleic Acids Res. 23, 3842- 3849.

12. Hoheisel, J . D. (1996) Nucleic Acids Res. 24, 430- 432.

13. Maier, T., and Pfleiderer, W. (1993) Collect. Czech. Chem. Commun. 58, 209- 213. 
14. Kierzek, R., Caruthers, M. H., Longfellow, C. E., Swinton, D., Turner, D. H., and Freier, S. M. (1986) Biochemistry 25, 78407846.

15. Sambrook, J ., Fritsch, E. F., and Maniatis, T. (1989) Molecular Cloning: A Laboratory Manual, Cold Spring Harbor Laboratory Press, Cold Spring Harbor, NY.

16. Maskos, U., and Southern, E . (1992) Nucleic Acids Res. 20, 16791684.

17. Matson, R. S., Rampal, J ., Pentoney, S. L., Anderson, P. D., and Coassin, P. (1995) Anal. Biochem. 224, 110- 116.

18. Albretsen, C., Kalland, K.-H., Haukanes, B.-I., Håvarstein, L.-S., and Kleppe, K. (1990) Anal. Biochem. 189, 40- 50.
19. Stengele, K. P., and Pfleiderer, W. (1990) Tetrahedron Lett. 31, 2549-2552.

20. Weiler, J ., and Pfleiderer, W. (1995) Nucleosides Nucleotides 14, 921- 924.

21. Weiler, J ., and Pfleiderer, W. (1996) in Innovations and Perspectives in Solid Phase Synthesis and Combinatorial Chemical Libraries (Epton, R., Ed.), Mayflower Worldwide, Edinburgh, in press.

22. Brown, T., Pritchard, C. E., Turner, G., and Salisbury, S. A. (1989) J . Chem. Soc. Commun., 891-893.

23. Katzhendler, J ., Cohen, S., Rahamim, E., Weisz, M., Ringel, I., and Deutsch, J . (1989) Tetrahedron 45, 2777-2792.

24. Pirrung, M. C., and Bradley, J .-C. (1995) J . Org. Chem. 60, 62706276. 